ON

\title{
DISARTICULATION OF THE SCAPUL $\Lambda$
}

\author{
FROM THE SHOULDER-JOINT.
}

\author{
BY
}

JAMES SYME, F.R.S.E.

PROFESSOR OF CLINICAL SURGERY IN THE UNIVERSITY OY EDINBURGH.

Received Jan. 3d.-Read Feb. 24th, 1857.

Having lately had occasion to perform an operation, which, so far as I am aware, is new in the practice of surgery, I venture to hope that some account of it may not be deemed unworthy of attention by this Society, in whose 'Transactions' are recorded so many additions to the resources of surgical art.

Janet Scott, according to her own account, about sixty, but as it was afterwards ascertained, nearly seventy years of age, was admitted into the Royal Infirmary, on the 18th of September last, on account of a large tumour involving the left scapula. In size and form it resembled a cocoa-nut, its consistence in some parts presented the hardness of bone, and in others was elastic but firm. A distinct aneurismal bruit could be perceived throughout the swelling, which also communicated a strong pulsatory movement to the hand when placed over it. The patient stated that she had first noticed the enlargement about six months before the time of her application to me, when it was the size of an orange, and had suffered little inconvenience from it until a recent period, when, not only from rapidly increasing, but also from causing painful sensations, it had 
rendered her unable to discharge her duties as a domestic servant in the country.

As the growth extended into the axilla, it was evidently impossible to afford relief by any partial removal of the scapula, and as amputation of the arm, together with the tumour, independently of the objection that might be expected from the patient to so fearful a mutilation, appeared altogether hopeless of success at her advanced time of life, I took into consideration the practicability of disarticulating the affected bone alone. The tumour, if not aneurismal, was evidently very vascular, and I knew from the previous experience of partial operations that the removal of the whole bone could not be accomplished without a formidable amount of hxmorrhage. On the other hand, I thought there was reason to expect that, if the subscapular artery were secured at an early part of the process, the clavicular and humeral attachments might be separated without much loss of blood, and that then the remaining connections would admit of such rapid division as to place the cut vessels almost immediately within reach of compression. It also appeared probable that, if the object were thus accomplished without an excessive drain on the patient's strength, there would not be any other serious obstacle to recovery, or threatening source of danger, and I therefore resolved to perform the operation.

On the 1st of October, the patient being fully under the influence of chloroform, and placed upon her right side, I made an incision from the acromion process transversely to the posterior edge of the scapula, and another from the centre of this one directly downwards below the lower margin of the tumour. The flaps thus formed being reflected without much hæmorrhage, I separated the scapular attachment of the deltoid, and divided the connections of the acromial extremity of the clavicle. Then, wishing to command the subscapular artery, I divided it with the effect of giving issue to a fearful gush of blood, but fortunately caught the vessel and tied it without any delay. I next cut into the joint and round the glenoid cavity, hooked my 
finger under the coracoid process so as to facilitate the division of its muscular and ligamentous attachments, and then pulling back the bone with all the force of my left hand, separated its remaining attachments with rapid sweeps of the knife. The vessels requiring ligature having been tied, the edges of the wound were stitched together and covered with dry lint, a bandage being lastly applied round the chest to give proper support, and keep the arm in its place.

The tumour, when examined, was found to consist of a nearly uniform expansion of the bone into a bag, partly membranous, partly osseous, containing a soft very vascular growth of the cerebriform kind. It may be seen from the preparation before the Society, that this condition, not only extended up to the spine of the scapula, but also implicated the margin of the glenoid cavity, so that any operation which did not reach the joint could not have extirpated the whole existing disease.

Everything went on favorably after the operation, and a great part of the wound healed by the first intention. The discharge, which was at first rather copious and thin, in the course of a few days diminished to an amount so small, as to remove all apprehension of its exhausting the patient, especially as she retained a good appetite, even for the porridge which had been her principal article of diet previously, slept soundly, and presented a cheerful aspect. At the end of a fortnight the discharge was reduced to little more than sufficient for staining the bandage, so that it seemed as if complete recovery would very soon be accomplished. But through an opening about an inch in length, where the edges of the transverse incision remained ununited, the head of the humerus could be seen still covered with its cartilage, which, however, in the course of another week began to disappear, and to give place to granulations gradually extending from the neck over the convexity of the bone; while the cavity at the same time contracted until the humerus and clavicle came nearly in contact, and the shoulder, especially when viewed in front, assumed a wonder- 
fully natural appearance. The patient, who from an early period after the operation had with difficulty been restrained from using the arm too freely, again and again declared that it was in no wise inferior to the sound one, and it appeared, indeed, that through the support afforded by the clavicular portion of the deltoid, together with the action of the pectoralis and latissimus dorsi, the limb would be able to execute a fair degree of motion.

While the local state of matters was thus proceeding in the most favorable and satisfactory manner, it could not escape observation that the patient's strength did not improve in a corresponding degree. On the contrary, without any reason that could be discovered except old age, she gradually became weaker and more emaciated, though still retaining appetite for food, and performing her bodily functions with so much appearance of health as still to encourage the hope of ultimate recovery. But towards the end of November symptoms of sinking suddenly presented themselves, and terminated in death on the 1st of December.

Although it would have no doubt been more satisfactory if the patient had lived longer, it is evident that the progress of her case was sufficiently advanced for the settlement of some points possessing practical importance. It thus appears, in the first place, that the entire scapula may be disarticulated from the shoulder-joint without a loss of blood to any great extent ; 2dly, that the wound resulting from this operation does not necessarily occasion an excessive amount of discharge; and, 3dly, that the arm which remains is not a useless appendage, but a serviceable limb. The value of these facts will of course depend upon the field admitting of their application to practice ; and the extent of this can hardly be determined at present, since morbid conditions, involving the whole scapula having been hitherto regarded as irremediable, may not have seemed deserving of record. One remarkable case, which would have afforded an excellent opportunity of performing the operation, is strongly impressed upon my memory, and may not be unknown to some members of the Society. I allude to that 
of the boy whose history Mr. Liston has related in his 'Elements of Surgery,' and more fully in the 'Edinburgh Medical and Surgical Journal' for 1820. The patient, a youth sixteen years of age, had come from the country on account of a tumour involving the scapula, and been dismissed from the Royal Infirmary as incurable. He was then placed under the care of Mr. Liston, who, finding that the growth was confined to the bone, and that it moved freely over the ribs, resolved to operate. The tumour, which was about the size of an orange, had been first perceived about three months before, situated immediately below the spine of the scapula, not larger than a filbert, of a flat form, and attended with distinct pulsation. It subsequently increased with great rapidity until it extended from the inferior angle over two thirds of the scapula. It was uniformly convex, and possessed a very firm consistence, but yielded under strong pressure with a crackling sensation.

The external surface of the tumour was exposed without any difficulty; but when the operator attempted to separate its attachment to the spine of the scapula, such a gush of blood issued as to require the most energetic measures of suppression. The subscapular and other vessels concerned in this hæmorrhage having been secured, Mr. Liston sawed across the scapula so as to leave merely its upper portion to the extent of about a third part of the spine. The tumour was found to consist of an osseous shell composed of plates directed from the circumference towards the centre, and containing a coagulum of blood in its cavity. It has generally been regarded as an example of osteo-aneurism, but I believe was rather, a form of cerebriform disease. Notwithstanding the patient's extreme exhaustion during and immediately after the operation, everything went on favorably until between five and six weeks, when a fungous excrescence appeared at the upper part of the wound. This was removed so as to expose the bone and allow it to be cauterized; but resisted the check thus opposed to its growth, and showed that so long as any portion of the scapula was permitted to remain, there could not be any 
reasonable expectation of recovery. Mr. Liston therefore proposed to remove the part which had been left at the former operation, together with the arm, but could not obtain the sanction of any surgeon in Edinburgh for such a formidable proceeding, and did not feel warranted to undertake it upon his own responsibility. He then, as a last resource, applied to Dr. Barclay, who was a most energetic and successful teacher of anatomy, but had never been engaged in professional practice. At the consultation which now took place, all the arguments in favour of interference, such as the hopelessness of the case, the youth of the patient, and the sort of precedent afforded by Cheselden's remarkable history of Samuel Wood, the miller, who recovered after his arm had been torn off along with the scapula, were met by one insuperable objection, which was the danger, or rather, as it seemed, certainly fatal effect that must attend division of the great blood-vessels so near the heart. Nothing therefore was done, and the patient, after lingering in great misery and being finally exhausted by repeated hæmorrhages, died five months after the operation.

In this case the practicability of disarticulating the scapula from the shoulder joint would have been a most welcome suggestion, and prevented much painful as well as never-ceasing regret by affording means of relief not exposed to the objections which prevented adoption of the formidable mutilation that had been proposed as the only mode of remedy. I may add that knowledge of the independence of the arm in regard to mobility on the presence of the scapula will tend to encourage greater freedom in operating for disease of the shoulder joint than has hitherto been practised. Upon these grounds I venture to hope that the communication which has now been offered to this Society may prove not entirely useless, and that although the operation did not lengthen the life of the patient directly concerned, it may become the means of doing so under other circumstances more favorable for complete and permanent recovery. 